\title{
Hechos estilizados del mercado de criptomonedas desde el enfoque de la eficiencia marginal del capital.
}

\section{Stylized facts of the cryptocurrency market from the perspective of the marginal efficiency of capital.}

Armando José Urdaneta Montiel. ${ }^{1}$, Emmanuel Vitorio Borgucci García. ${ }^{2}$, John Alexander Campuzano Vasquez. ${ }^{3} \&$ Pedro Alexander Avilés Almeida ${ }^{4}$.

DOI: https://doi.org/10.33262/visionariodigital.v3i2.421

\section{Resumen.}

El presente estudio tiene como objetivo analizar el mercado de criptomonedas desde el enfoque de la teoría de la demanda de activos y dentro de ella de los retornos esperados, tomando en cuenta dos de las más importantes: el Bitcoin (minada) y el Ripple (no minada). En esta investigación exploratoria, analítica y descriptiva, se tomó en cuenta el precio del cierre, volumen de transacciones, capitalización en el mercado y cantidad de criptomonedas emitidas, utilizando las variaciones diarias en términos porcentuales. Los resultados obtenidos muestran que las monedas consideradas de acuerdo a sus variaciones de precios de cierre diario y cantidad de criptomonedas emitidas, evidencian una evolución que se ajusta a la ley de los rendimientos marginales decrecientes unos de los fundamentos de la teoría eficiencia marginal del capital; mientras en el caso de las variaciones diarias de la capitalización del mercado con respecto a las de criptomonedas emitidas, comportan una evolución creciente, inversamente a los precios de cierre.

Palabras clave: Criptomonedas, bitcoin, mercado, monedas
Abstract
The objective of this study is to analyze the cryptocurrency market from the perspective of the theory of the demand for assets and within it the expected returns, taking into account two of the most important: the Bitcoin (mined) and the Ripple (no mined). In this exploratory, analytical and descriptive investigation, the closing price, volume of transactions, capitalization in the market and number of cryptocurrencies issued were taken into account, using the daily variations in percentage terms. The results obtained show that the currencies considered according to their variations of daily closing prices and the amount of cryptocurrencies issued, show an evolution that adjusts to the law of
1 Docente Universidad Metropolitana, Sede Machala, Ecuador, aurdaneta@umet.edu.ec
2 Docente Universidad del Zulia, Maracaibo, Venezuela, emmanuelborgucci@gmail.com
3 Docente Universidad Técnica de Machala, Ecuador, jcampuzano@utmachala.edu.ec
${ }^{4}$ Docente Universidad de Guayaquil, Ecuador, avilesa@ug.edu.ec 
diminishing marginal returns one of the foundations of the marginal efficiency theory of capital; while in the case of the daily variations of market capitalization with respect to those of cryptocurrencies issued, they show an increasing evolution, inversely to the closing prices.

Keywords: Cryptocurrencies, bitcoin, market, coins

\section{Introducción.}

En el mundo de hoy surge una nueva oportunidad de inversión y ahorro para generar ingresos sustentados en la tecnología blockchain, que definitivamente está cambiando la forma de hacer negocios y comercializar bienes y servicios a través de monedas electrónicas o virtuales minadas como el Bitcoin y no minadas como el Ripple. A las criptomonedas se las considera en los actuales momentos como una de las inversiones más rentables y más seguras, ofrecen un abanico de posibilidades y portafolios de inversión que ayudan maximizar la rentabilidad esperada del dinero destinado a su adquisición, con la posibilidad de ganar dinero las 24 horas del día. Pero también las criptomonedas responden a un nuevo tipo de economía política que representa un desafío a los Estados nacionales ${ }^{5}$.

Sin embargo, es necesario conocer la existencia de diferencias entre el dinero virtual y los medios de pago tradicionales, y las transacciones realizadas en esta red de tecnología Blockchain, hechos que en los actuales momentos motivan su uso por su no rastreabilidad y comportamiento anónimo, así como la completa ausencia de intermediarios, transacciones que una vez hechas no pueden reversarse (Rogojanu \& Badea, 2014). Una particularidad que causa polémica alrededor de este tipo de monedas, es que ya están siendo utilizadas para comercio ilegal como tráfico de drogas, prostitución infantil, robos apuestas ilegales, entre otros (Krugman, 2013).

Otro aspecto negativo y de desconfianza sobre la inversión de criptoactivos a largo plazo, es que no tiene respaldo de ningún Estado o Banco Central de ningún país (Guttman, 2014). Esta característica obstaculiza su regulación, cobro de impuestos y el consiguiente temor de Estados y bancos, a perder el monopolio de la emisión monetaria, por lo que algunos países prohíben su uso.

Por ello el objetivo de la presente investigación consiste analizar el mercado de criptomonedas desde el enfoque de la eficiencia marginal del capital, en aras de develar la relación existente entre el índice de variación de capitalización del mercado sobre el precio de cierre de la criptomoneda, así como, los hechos que se vinculan a su uso y expansión en el mercado de activos a nivel mundial.

\footnotetext{
${ }^{5}$ El Manifiesto del Nuevo Libertarianismo, Samuel Konkin III (1980) expone lo que debería ser una economía cuyo desarrollo no dependa del Estado. El manifiesto sugiere: 1. Los seres humanos son coercionados; 2. la coerción es inmoral, ineficiente e innecesaria para la vida humana; 3. las instituciones de la coerción están centralizadas en el Estado; 4 la coerción más fuerte es por medio de los impuestos y la inflación, que ha confiscado los ahorros de los ciudadanos emprendedores; y 5. la propaganda política y la instrucción pública ha instaurado mentiras más grandes que el mito presocrático. Entre sus premisas se encuentran: 1. la violencia del Estado y sus amenazas son dañinas, malas, inmorales, diabólicas y supremamente impracticable (Konkin III, 1980: 3); 2. es el Estado versus el Agorá; 3. investiga la naturaleza humana para explicar sus derechos y promover su aplicación; 4. busca combatir la "Casta intelectual corrupta" que adquiere las denominaciones de: progresistas, socialistas, revolucionarios, reformistas, minarquistas, la partidocracia, etc. (Konkin III, 1980: 3). De igual manera, surge el concepto de anarcocapitalismo y el criptoanarquismo. El primero fue impulsado principalmente por David Friedman, Rothbard Murray y Hans-Hermann Hope. Este anarquismo es de corte individualista y por esta razón se distancia del anarquismo colectivista de Joseph Proudhom. El segundo se muestra favorable de la criptografía asimétrica en aras de la libertad y la privacidad y fue un término popularizado por Timothy C. May y es la realización ciberespacial del anarcocapitalismo.
} 


\section{Desarrollo.}

Las criptomonedas las producen los mineros, que suelen ser programadores que tienen equipos informáticos de minado muy potentes como los ASIC (Circuito Integrado para Aplicaciones Específicas) que tiene como propósito resolver bloques blockchain. Siendo esta tecnología la principal garantía de seguridad del mercado de las criptomonedas. Debido a que las mismas no están bajo el control de una autoridad monetaria central (Rogojanu \& Badea, 2014).

Las criptomonedas progresivamente se están convirtiendo en un medio de pago global, y por tanto, existe un creciente volumen de oferta y de demanda, conformando un mercado descentralizado y diversificado. Descentralizado, por cuanto las operaciones ocurren en cualquier lugar del mundo donde se acceda a sistemas de computación y de transmisión de datos; y diversificado, debido a la existencia de muchos tipos de criptomonedas y de criptoactivos a disposición de los clientes potenciales.

Este proceso de descentralización y diversificación está produciendo constantes fluctuaciones en su valor de cambio, bien sea con otra criptomoneda, o con una moneda de curso legal. Por ello se han convertido en una estrategia de inversión a corto plazo, más no así a largo plazo, debido sus recientes escenarios de volatilidad en precios. Sin embargo sigue existiendo una alta confianza por parte de los agentes económicos tenedores de criptoactivos, debido a los estándares de seguridad que brinda la tecnología blockchain, la cual les permite trascender los límites geográficos operando y gestionado desde cualquier parte del mundo (Draper, 2013) ${ }^{6}$.

El elemento de seguridad de las criptomonedas es lo que respalda el principal atractivo de este mercado cuyos precios aparentemente siguen la llamada "ley de la oferta y la demanda". Esto puede provocar fuertes subidas pero también grandes caídas. Por ello existen países como Rusia, China e Islandia, que no fomentan su uso, lo que evita llegar al máximo número de personas en el mundo (Laurence, 2017).

Hace apenas unos 15 años atrás, el diseño de medio de pagos creados al margen de los Estados eran un sueño. En ese sentido Yermack (2013) señala que la búsqueda de formas de dinero alternativo como las criptomonedas, surge a raíz de la desaparición del patrón oro en el año 1971, a partir de allí, la mayoría de las economías industrializadas y no industrializadas emiten papel moneda fiduciario, que se basa en la fe pública de que los gobiernos, a través de sus Bancos Centrales no aumentaran la oferta monetaria con demasiada rapidez.

\footnotetext{
${ }^{6}$ Todo ello mediante claves de seguridad, con sistemas de seguridad anónima para las personas, cuyos pagos se formalizan de manera instantánea, sin intermediarios, a través del escaneo del código QR de la persona que va realizar el pago e identificar la cantidad deseada, por lo que se puede realizar desde cualquier móvil con conexión a internet; donde los impuestos que incluye cada transacción son mínimos, así como las tasas para enviar dinero al exterior. Sumado ello está la privacidad de la que gozan los usuarios que las poseen y las tranzan, ya que no son públicos sus nombres, al contrario de lo que ocurre con las cuentas y tarjetas de crédito; aunque cabe destacar que todas las transacciones realizadas quedan registradas de forma permanente en el blockchain de cada criptodivisa, (Antonopoulos, 2015). Puede decirse entonces, que la principal desventaja es la necesidad de tener internet para acceder a las criptomonedas, caso contrario, no se puede disponer de ellas a través de los wallets y gestionarlo de ningún modo. En ese sentido una de las amenazas de las criptomonedas son las computadoras cuánticas, que aunque todavía no se encuentran en el mercado, representan serios riesgos para este tipo de monedas electrónicas por su capacidad de procesamiento, lo cual amenaza los sistemas que utilizan criptografía como método de seguridad (Norman, 2017). No obstante, para quienes utilizan estas monedas digitales, por su seguridad se hace necesario que realicen una copia de seguridad de sus wallets o monederos digitales, para evitar correr riesgo de perder todo su dinero; en ese sentido es fundamental el buen resguardo tanto de su clave pública y privada; las cuales son garantías con su tecnología blockchain de la realización de gran volumen de transacciones digitales con un alto grado de codificación que no poseen las transferencias bancarias comunes, registrando todas las operaciones en una base de datos, las cuales se certifican para que no se puedan duplicar o falsificar.
} 
Desde el punto de vista económico, el surgimiento de las criptomonedas acepta el principio de: "Que un cambio en el ambiente económico estimulará la búsqueda de innovaciones que generen ganancias" (Mishkin, 1995: 232). En este sentido, existen tres tipos básicos de innovaciones financieras: las que responden a cambios en las condiciones de demanda, las que responden a cambios en las condiciones de oferta y las que responden a las circunstancias de evadir las regulaciones gubernamentales.

Del lado de la demanda, las innovaciones comenzaron a aparecer debido a las fluctuaciones en el tipo de interés de las letras de tesoro estadounidense en la década de los años 50, 70 y 80 . Todas estas fluctuaciones representaron enormes pérdidas para los mercados de dinero y de capitales. Tres ejemplos de innovaciones financieras asociadas a la demanda, por ejemplo, fueron: las Adjustable-Rate Mortgages, los Financial Future Markets (con los future contracts o los financial future contracts) y los Financial Options Markets (con las call y put options). Estas innovaciones generaron mercados tales como: los Savings \& Loans Institutions, el Chicago Board of Trade (CBT) o Chicago Board Options Exchange (CBOT).

Por el lado de la oferta, se encuentra el formidable desarrollo de los sistemas de comunicación y los sistemas informáticos. Este desarrollo permitió, por ejemplo, el surgimiento de los procesos de Securitization (Mishkin, 1995) ${ }^{7}$. La Securitization: "Es el proceso de transformar activos financieros de baja liquidez, tales como hipotecas sobre viviendas, en títulos valores comercializables en los mercados financieros" (Mishkin, 1995). El proceso comenzó en la década de los 70 con la creación de los Mortgage-backed security en el mercado de las hipotecas y fue seguida con instrumentos similares en el mercado de los créditos para automóviles (Certificates of Automobile Receivables conocido como CAR o el First Automobile Short Term Bonds and Certificates o FASTBACs), letras del tesoro (Treasury Investment Growth Receips o TIGRs), los Certificates of Accrual on Treasury Securities (CATS). Finalmente, uno de los instrumentos más populares en los mercados financieros fueron los Collateralized Mortgage Obligations (CMO en adelante), que son bonos construidos sobre una cartera de hipotecas ${ }^{8}$.

Ahora bien, como el negocio financiero en general está fuertemente regulado por el Estado, esta regulación es una fuente para las innovaciones financieras. En otras palabras, la regulación genera incentivos no para evadir la regulación, sino para trabajar con ella es lo que Kane (1981) denominó como "Loophole minning". Así, las regulaciones detrás de las innovaciones financieras son, por ejemplo: las exigencias de reservas a las instituciones financieras, que imponen un costo para los bancos equivalente el tipo de interés que podían ganar si las reservas se dieran en créditos.

Por otra parte, se encuentran las restricciones sobre intereses pagados sobre los depósitos, mediante el establecimiento de techos tanto para los depósitos como para los créditos. Esta situación en los Estados Unidos; por ejemplo, condujo a un proceso de desintermediación bancaria y el surgimiento de un mayor volumen y tipos de instituciones

\footnotetext{
${ }^{7}$ Los desarrollos del lado de la oferta incluyen, por ejemplo: las tarjetas de crédito bancarias, el mercado de los Junk Bonds, el surgimiento del mercado de los Commercial Papers, la internacionalización de los mercados financieros.

${ }^{8}$ Con la ayuda de los sistemas de cómputo, las CMO podían dividirse en tranches clases. Los primeros tres cortes reciben los intereses de acuerdo a la tasa del cupón sobre el CMO. El primer corte recibe el capital y los prepagos de los colateralizados pool de hipotecas. Después de la primera clase (tranche), los pagos del capital los intereses y los prepagos se retiran de manera secuencial. La cuarta clase, llamada Accrual o clase Z (bonos de largo plazo) reciben el interés y el principal solamente después que las otras clases han sido liquidadas
} 
financieras no bancarias tales como: las aseguradoras, los fondos de pensión, las compañías financieras (Sales finance companies, Consumer finance companies o las Business finance companies), los Fondos Mutuales, los Fondos Mutuales del mercado de dinero, los Bancos de Inversión, las agencias federales de crédito (Federal National Mortgage Association o FNMA, el Government National Mortgage Association o GNMA o el Federal Home Loan Mortgage Company o FHLMC), los Farm Credit System, The Student Loan Marketing Association (Sallie Mae), Securities Brockers and Dealers o las Organized Exchanges (tales como la National Association of Securities Dealers Automated Quotation System o NASDAQ), entre otras instituciones.

La crisis de 2007, denominada de los Subprime y de las CMO, produjo un nuevo movimiento en la regulación, acomodándose el sistema en términos de la oferta, surgiendo un nuevo tipo de concepto financiero denominado criptomoneda. Del grupo de las denominadas criptomonedas, el Bitcoin es la más representativa de las monedas digitales minables y su protocolo fue publicado en el 2008 por Satoshi Nakamoto. El 3 de enero de 2009 comienza a funcionar la red P2P de los Bitcoins (Reid \& Harrigan, 2013). Mientras que dentro de las monedas digitales no minables se encuentra el Ripple, la cual entra en cotización el 4 de agosto de 2013, la red de pago RippleNet y su token de liquidación utilizado por AMEX hace que sea conocida como la moneda digital de los grandes bancos.

Un activo, desde el punto de vista económico es un objeto en poder de un propietario y que es una reserva de valor. Bienes como bonos, edificios, tierras, fábricas o fincas y otros más se les considera activos. La adquisición, la venta o la retención de cualquier activo estará sujeto, según Markowitz (1952, 1970), Roy (1952), Hicks (1962) o Sharpe (1963), a las siguientes consideraciones: 1) la riqueza que es total de recursos propiedad de un individuo, grupo de ellos o de empresas; 2) los retornos esperados o el rédito que el activo pudiese generar con relación a otros activos; 3) el riesgo o el grado de incertidumbre asociada con el retorno esperado de un activo con relación a otros activos; y 4) la liquidez del activo o la capacidad de convertirse fácilmente en efectivo al menor descuento posible con relación a activos alternativos.

En el caso específico de los retornos esperados, un activo será más deseado que otro debido a, por lo menos, dos factores: cuando el retorno esperado del activo A crece frente al retorno esperado en el activo alternativo, que permanece constante; y cuando el retorno del activo alternativo cae frente al retorno esperado en el activo A. de manera más general, el retorno esperado es equivalente a la sumatoria ponderada de cada posible retorno realizado multiplicado por la probabilidad de ocurrencia:

$$
R E T^{e}=\sum_{i=1}^{n} \text { Pi.RETi }
$$

Donde:

RET $^{\mathrm{e}}$ : es el retorno esperado

Pi: la probabilidad de realización del RETi

RETi: el retorno realizado 
Cuando, por ejemplo, un individuo, empresa o fondo de pensión adquieren un activo son acreedores a una serie de rendimientos probables hasta el momento que vende o termine la vida útil del activo adquirido. Esos rendimientos son una serie de anualidades RET $^{1}$, $\mathrm{RET}^{2}, \ldots \mathrm{RET}^{\mathrm{n}}$ que es el rendimiento probable de la inversión (Keynes, 1981). En este sentido Keynes (1981) diferencia entre el precio de oferta del activo (el precio que exactamente haría que el poseedor del activo adquiera una nueva unidad adicional) y el rendimiento probable (precio de demanda). El primero es el conjunto de rendimientos previstos descontados por la eficiencia marginal del capital y el segundo la sumatoria de los rendimientos previstos descontados al tipo de interés 9 .

En este sentido, el precio de oferta será el siguiente:

$$
\text { Precio de Oferta }=\sum_{=1}^{n} \frac{R E T i}{(1+e m c)}
$$

Donde emc es la eficiencia marginal del capital o el tipo de interés que igualaría el precio de oferta con los rendimientos esperados descontados a valor presente. Por otra parte, el precio de demanda sería expresado así:

$$
\text { Precio de demanda }=\sum_{=1}^{n} \frac{R E T i}{(1+i)}
$$

Donde " $i$ " es el tipo de interés de mercado e indica el rendimiento futuro esperado descontados al tipo de interés y constituye su verdadero valor presente, lo que vale el activo en el mercado. De esta manera si el precio de demanda crece es debido a que el tipo de interés de mercado cae. Cuando el tipo de interés es menor que el tipo de la eficiencia marginal del capital, será adecuado adquirir el activo.

Desde el punto de vista de las criptomonedas, si el tipo de interés en el mercado es mayor que la eficiencia marginal del capital asociado a las criptomonedas, entonces será lucrativo dedicar dinero para su adquisición, ya que el rendimiento esperado será mayor que su precio de oferta.

De esta manera, los rendimientos previstos del capital invertido, los cuales en el caso del mercado de las criptomonedas dependerán de sus expectativas de rendimientos previstos $^{10}$ y no por los rendimientos efectivos. Los rendimientos previstos serán una expresión de la productividad marginal de la inversión realizada en este caso en criptomonedas, que Blanchard et al (2006) consiste en la variación de la rentabilidad obtenida sobre cada unidad adicional de capital invertido respecto a la inversión.

\footnotetext{
${ }^{9}$ Aunque Keynes (1981) empleó la eficiencia marginal del capital para dar cuenta de activos de capital, consideramos que es aplicable a cualquier tipo de activo del cual se espera rendimientos esperados en el tiempo.

${ }^{10}$ Estas expectativas pueden ser de corto plazo y de largo plazo. Las primeras tienen cierto grado de estabilidad, por cuanto los resultados obtenidos en el pasado reciente son una orientación para el futuro próximo. Las de largo plazo son más inestables, ya que no dependen del pasado inmediato y dependen de muchos factores de carácter sistemático. Los agentes económicos, se enfrentan con básicamente con dos fuerzas opuestas: el ser adverso al riesgo y la necesidad de sobrevivir con ganancias. Esas fuerzas están representadas por el espíritu de empresa y la especulación (Keynes, 1981: 144). La primera hacer referencia a la previsión de los rendimientos probables de los bienes por todo el tiempo que duren y la especulación es la actividad de prever la psicología del mercado. Las previsiones a largo plazo son originadas más en las previsiones de los especuladores. Estas previsiones, a su vez, se sustentan en opiniones convencionales predominantes.
} 
En ese sentido la productividad marginal del capital puede ser creciente pues con un incremento de una unidad en el factor capital aumentará más que proporcionalmente su rendimiento. Si el crecimiento porcentual del factor capital fuese superior al de su rendimiento por cada unidad adicional invertida, la productividad marginal se consideraría decreciente, según Samuelson \& Nordhaus (2006), tal como se muestra en la figura 1 de las criptomonedas Bitcoin y Ripple en los últimos 3 años.

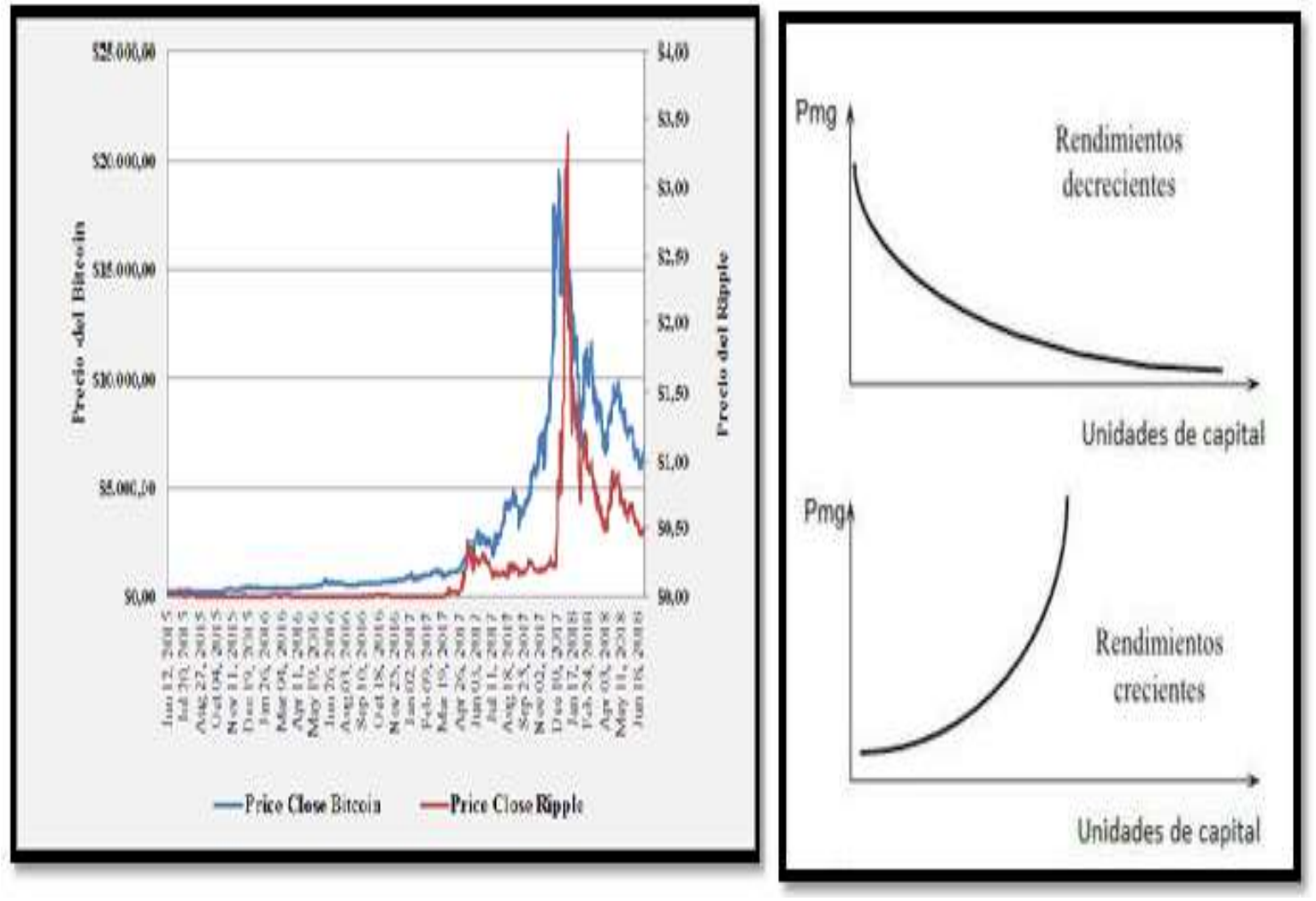

Figura 1. Cotización de las criptomonedas Bitcoin y Ripple 2015-2018 Fuentes: www.coinmarketcap

En la figura 1, puede observarse como ambas criptomonedas analizadas muestran una productividad marginal creciente desde junio de 2015 hasta diciembre de 2017, donde alcanzaron su máximo nivel de precios, para luego desplomarse en sus precios durante el primer semestre del año 2018, exhibiendo una productividad marginal decreciente. Todo ello viene dado la tasa de crecimiento de los precios, la cual estimaremos matemáticamente para el caso del presente estudio con su función total y su derivada, tomado en cuenta las cotizaciones de los últimos 36 meses.

Todo lo antes planteado esta también fundamentado en la ley de los rendimientos marginales decrecientes, la cual según Samuelson \& Nordhaus (2006), es la disminución del incremento marginal del capital a medida que se aumenta la inversión en este caso en la compra de criptoactivos o equipos de minado, y se aumenta la cantidad de Bitcoins o Ripples en circulación su precio tiende a disminuir al igual que su capitalización en el mercado y el volumen de comercio realizado en la compra y venta de criptoactivos. 
Este fenómeno también pudiera explicarse a través de la ley de utilidad marginal decreciente, según la cual el consumo de un bien proporciona menor utilidad adicional cuanto más se consume, manteniendo el consumo de los otros bienes constantes. Por lo que se produce la valoración decreciente del bien a medida que se consume una nueva unidad de este bien (Huerta de Soto, 2004). Comprar un criptoactivo adicional luego de satisfecha una necesidad, representa para el agente económico una utilidad inferior a la que representaba ese mismo bien cuando su demanda no estaba satisfecha.

\section{Método.}

El presente estudio utiliza métodos cuantitativos y estadísticos, basándose en fenómenos observables susceptibles de medición y análisis matemáticos Guerrero \& Guerrero (2014). Además, la investigación es analítica porque tienen como objetivo analizar un evento y comprenderlo en términos de sus aspectos menos evidentes. También, es una investigación exploratoria, porque el estudio permite hacer una aproximación a un fenómeno desconocido con el fin de aumentar el grado de conocimiento sobre el mismo, y aportar nuevas ideas sobre la evolución sobre el fenómeno objeto de exploración (Hernández et al, 2014).

Finalmente, el estudio es descriptivo porque detalla situaciones y eventos de cómo se manifiesta el objeto de investigación, en aras de especificar características importantes de sus parte componentes para medir su evolución (Bernal, 2006). En el trabajo se emplearon fuentes secundarias de datos, mediante las cotizaciones diarias de la capitalización del mercado, el precio de cierre de las criptomonedas y el volumen de transacciones comerciales, en las páginas web www.coinmarketcap.com, www.blockchain.com/es/charts_y https://es.investing.com/crypto/ripple/markets, con datos de los tres últimos años, específicamente en el período 12/06/2015 al 07/07/2018.

\section{Resultados y Discusión.}

En los resultados del presente estudio se manejan tres variables: la capitalización del mercado, el precio de cierre de las criptomonedas y el volumen de transacciones comerciales bien sea para el pago de bienes y servicios con criptomonedas o para el cambio por dinero convencional, tanto en la minada el Bitcoin, como en la no minada el Ripple, en aras de determinar su relación y evolución. Siendo la capitalización del mercado según Pérez (2011), el valor que tiene una compañía en el mercado de valores, en el caso de la criptomonedas sería el valor total de la inversión realizada en determinadas monedas digitales emitidas en el mercado de criptoactivos, su cálculo es muy sencillo, el cual consistiría en multiplicar el precio actual de una acción (precio de cierre diario de la moneda digital) por el número de títulos en circulación de la empresa (cantidad de monedas emitidas).

La capitalización del mercado de acuerdo con Ramirez (2007), presenta una fluctuación constante, debido a que el precio de cotización sufre variaciones a lo largo del periodo que el mercado está abierto, por tanto la capitalización bursátil varía en función del precio de cotización. Por ello la capitalización del mercado es uno de los criterios fundamentales a la hora de construir índices bursátiles que se ponderan por capitalización en función de la capitalización total del mercado. 


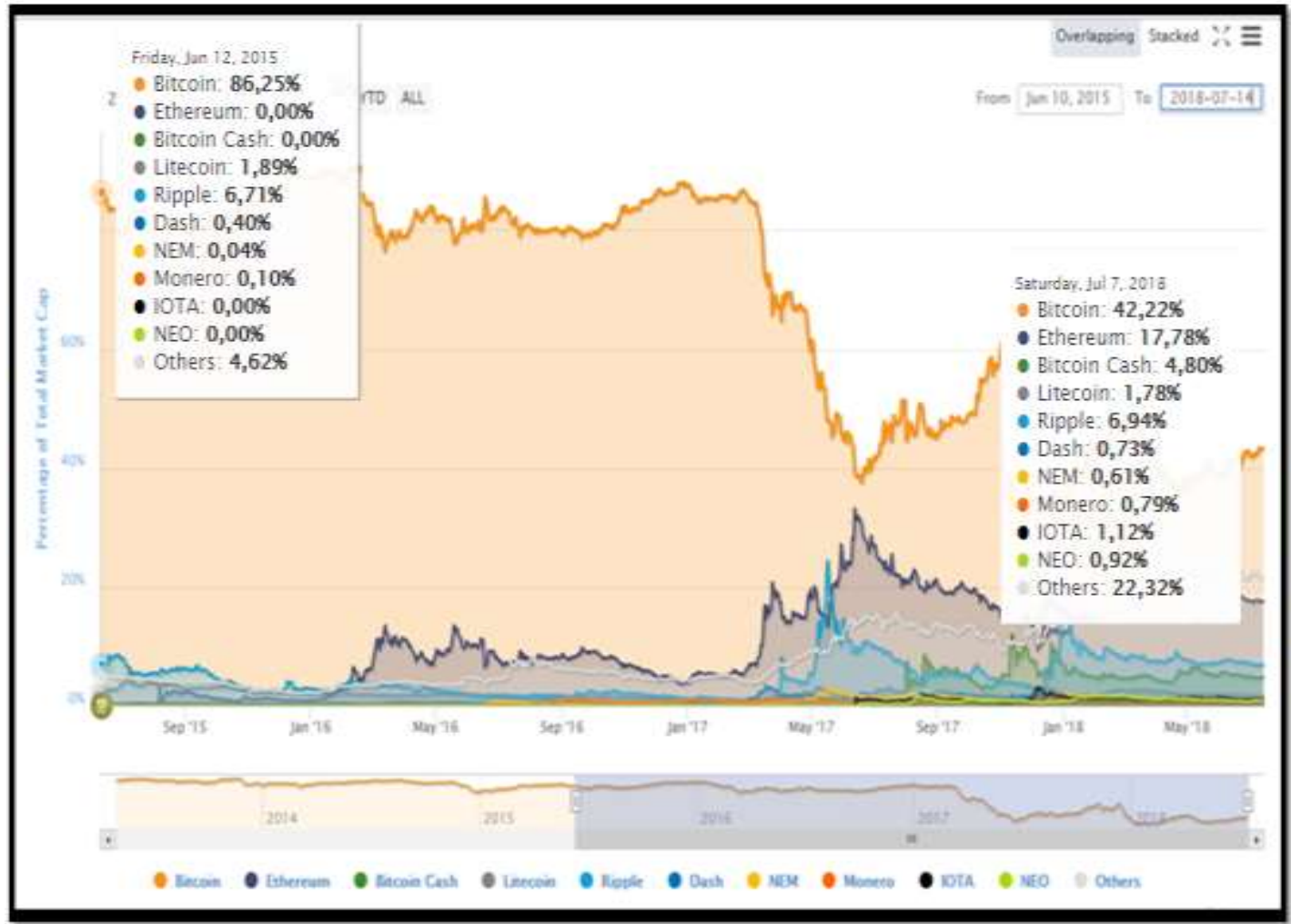

Figura 2. Capitalización total del mercado de la criptomonedas Fuente: www.coinmarketcap.com

En la figura 2, puede observarse como la criptomoneda Bitcoin ha perdido participación en el mercado de las criptomonedas, pasando del $86,25 \%$ de la capitalización total en junio-2015 a 42,22\% en Julio-2018, a pesar de que valor total de capitalización se ha incremento en el periodo de 3387,73\%. Mientras que el Ripple ha mantenido su participación un leve crecimiento pasando de $6,71 \%$ en junio de 2015 a $6,94 \%$ en julio de 2018; ello debido principalmente al bajo precio de dicha criptomoneda, en contraposición al Bitcoin cuyo precio mantiene un promedio mucho más elevado de precios.

No obstante, dichas criptomonedas muestran una evolución muy similar de como la variación del precio de cotización se trasmite a su capitalización en el mercado con un rezago intertemporal de 24 horas con respecto al precio de cierre como se muestra a continuación en los gráficos, 2, 3,4, y 5; donde se observa una mayor volatilidad en las monedas digitales no minadas que en las minadas, sobre todo en el último año del lapso estudiado.

En el caso del Bitcoin con un crecimiento promedio del precio diario del 0,38\% y del precio en el periodo estudiado de 2882,53\%; en cuanto a la capitalización del mercado un aumento promedio del valor diario del $0,38 \%$ y de capitalización en el periodo estudiado del 3387,73\%. Mientras en el caso del Ripple con un incremento promedio del precio diario del $0,69 \%$ y de dicho indicador en el periodo estudiado de $6012,41 \%$; en cuanto a la capitalización del mercado un aumento promedio del valor diario del $0,71 \%$ y de capitalización en el periodo estudiado del $7223,71 \%$.

Lo antes descrito se debe fundamental al crecimiento superlativo de la inversión en el mercado de las criptomonedas entre los dos últimos trimestres de 2017 y el primer 
trimestre de 2018, con el ingreso de nuevas criptomonedas al mercado y las bifurcaciones de las ya existentes o fork de blockchain, el cual consiste en la separación de cadenas blockchain, a partir de un código fuente principal de una criptomoneda existente, convirtiéndose en un nuevo proyecto de creación de una nueva cadena de bloques blockchain (nueva criptomoneda) a partir de una cadena principal, todo con la finalidad de captar más mercado con un precio mucho menor al de la moneda digital bifurcada previamente cuyo precio ha alcanzado valores muy altos restringiendo con ello la posibilidad a pequeños o noveles inversores de criptoactivos, el ejemplo más evidente son Bitcoin cash, Bitcoin Gold que son bifurcaciones de Bitcoin.

Lo cual lleva a corroborar la hipótesis de que el aumento del capital necesario a invertir para adquirir criptomonedas, restringe su demanda incidiendo negativamente en su participación en el mercado y finalmente en sus precios, provocando que quienes hayan invertido previamente vean que la eficiencia marginal del capital invertido sea cada vez menor (decreciente) y por ende su productividad o utilidad marginal. En razón de ello surge la estrategia entonces de bifurcar la moneda digital principal para no dejar de captar nuevos inversores y disminuir la presión sobre el nivel de demanda.

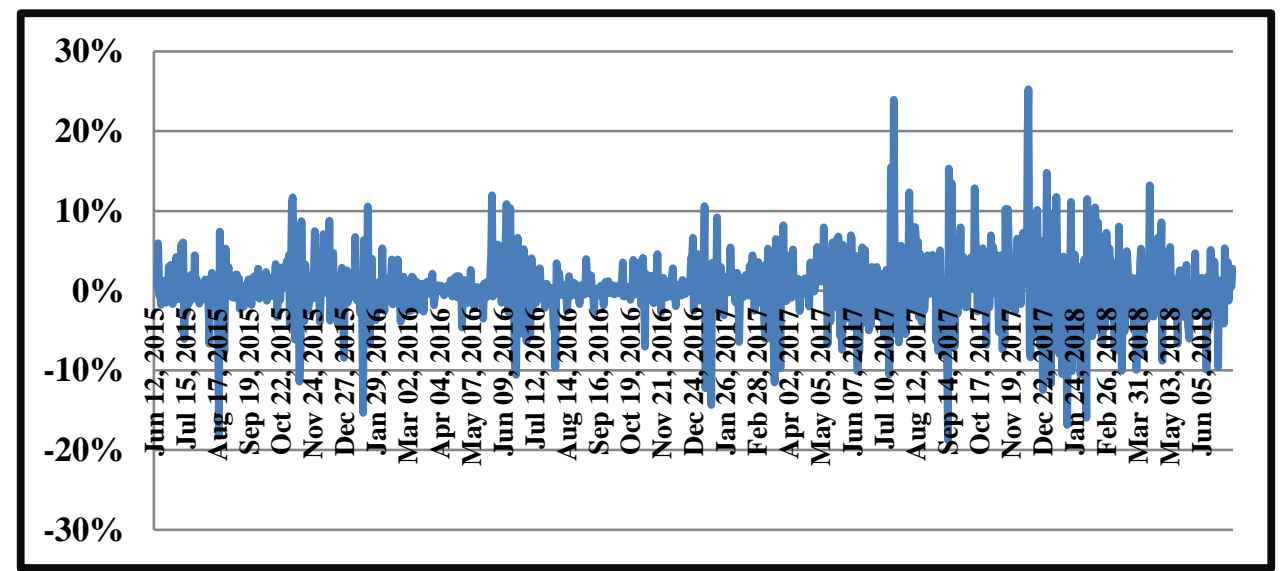

Figura 3.Variación del precio de cierre diario del Bitcoin

Fuente: Elaboración propia en base a datos de coinmarketcap.com

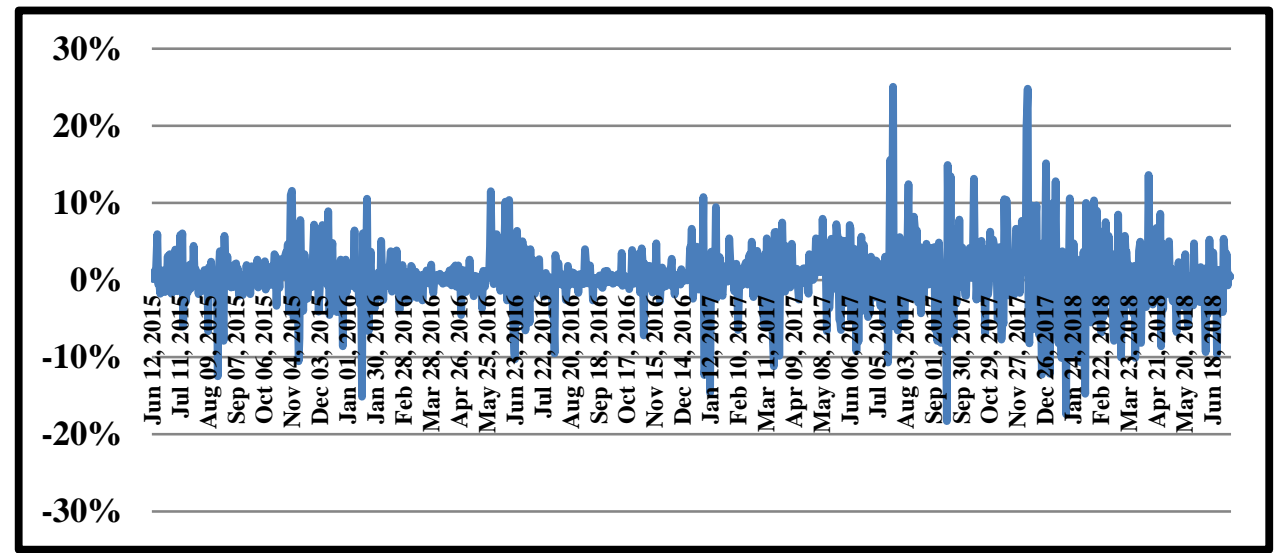

Figura 4.: Variación de la capitalización del mercado diario del Bitcoin Fuente: Elaboración propia en base a datos de coinmarketcap.com 


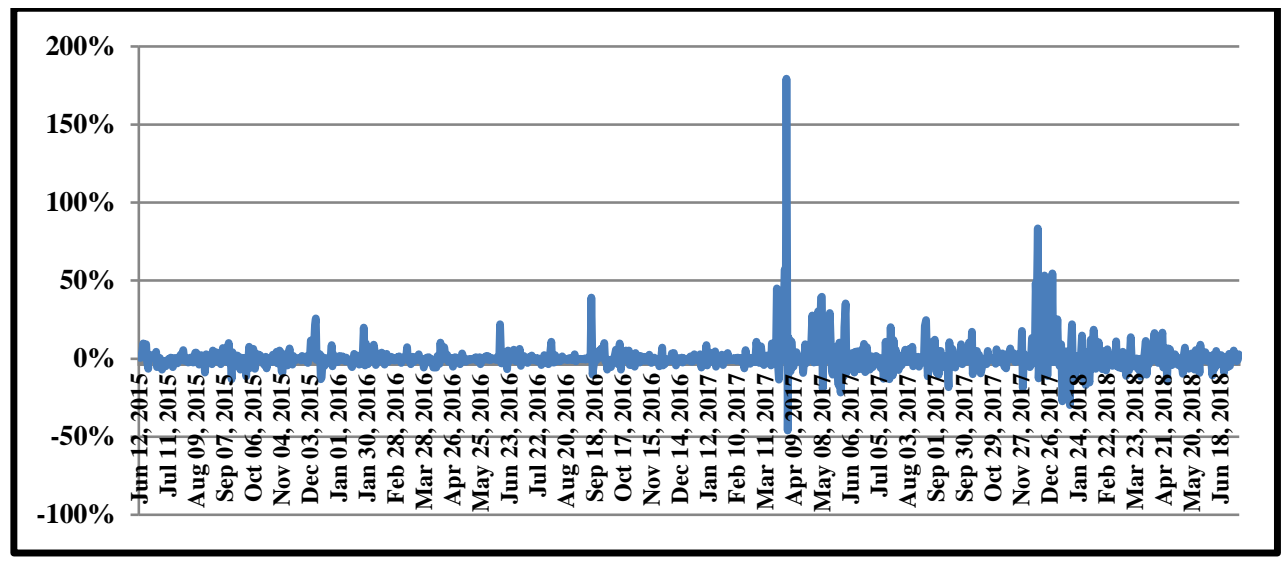

Figura 5. Variación del precio de cierre diario del Ripple

Fuente: Elaboración propia en base a datos de coinmarketcap.com

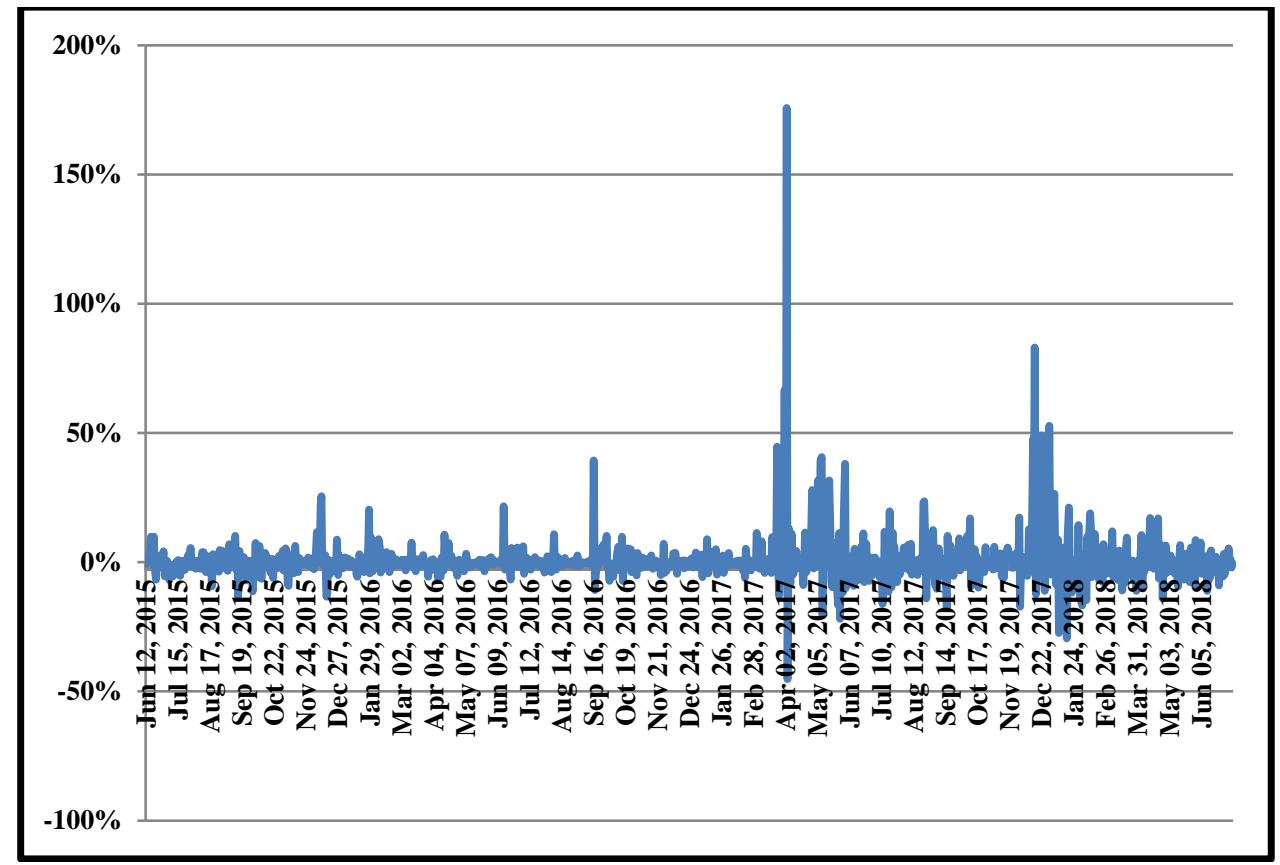

Figura 6. Variación de la capitalización del mercado diario del Ripple Fuente: Elaboración propia en base a datos de coinmarketcap.com

Como se señaló anteriormente, la capitalización en el mercado de una empresa se obtiene multiplicando el precio de sus acciones por el número de ellas, en el caso de la criptomonedas minadas y no minadas sujetas a estudio se cuenta con el valor de su capitalización de mercado y precio de cierre diarios; por ello al dividir la primera entre la segunda se obtendría una aproximación racional al número de criptomonedas emitidas (indicador bursátil) desde el punto de vista de la racionalidad bursátil de los mercados financieros; para posteriormente medir su variación porcentual diaria (índice bursátil), con respecto a la variación porcentual diaria del precio de cierre y la capitalización del mercado, en aras de constatar su evolución, como se muestra en figuras 7 y 8 . 


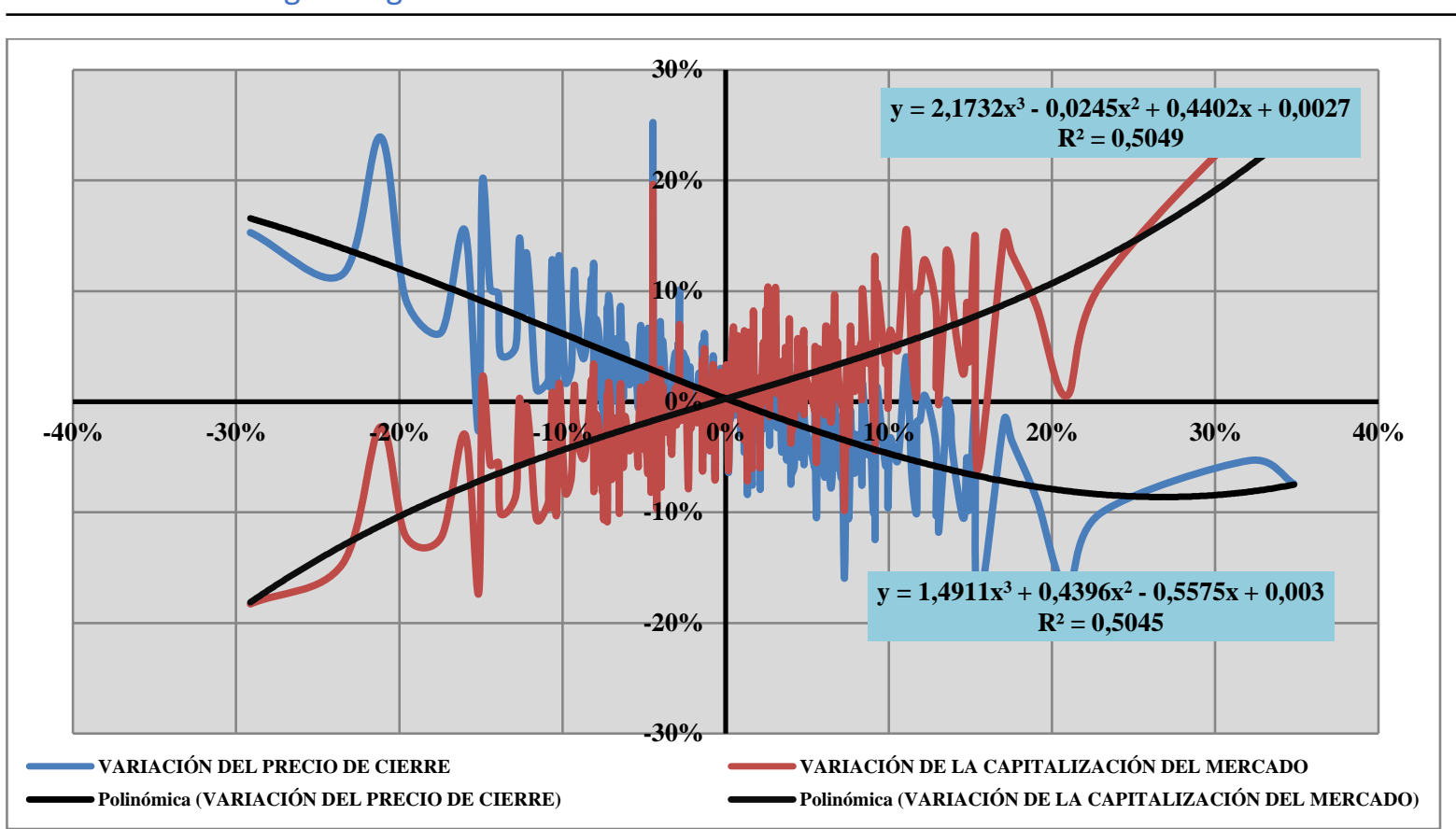

Figura 7.: Variación del indicador bursátil (índice bursátil) respecto al precio de cierre y la capitalización del mercado del Bitcoin

Fuente: Elaboración propia en base a datos de coinmarketcap.com

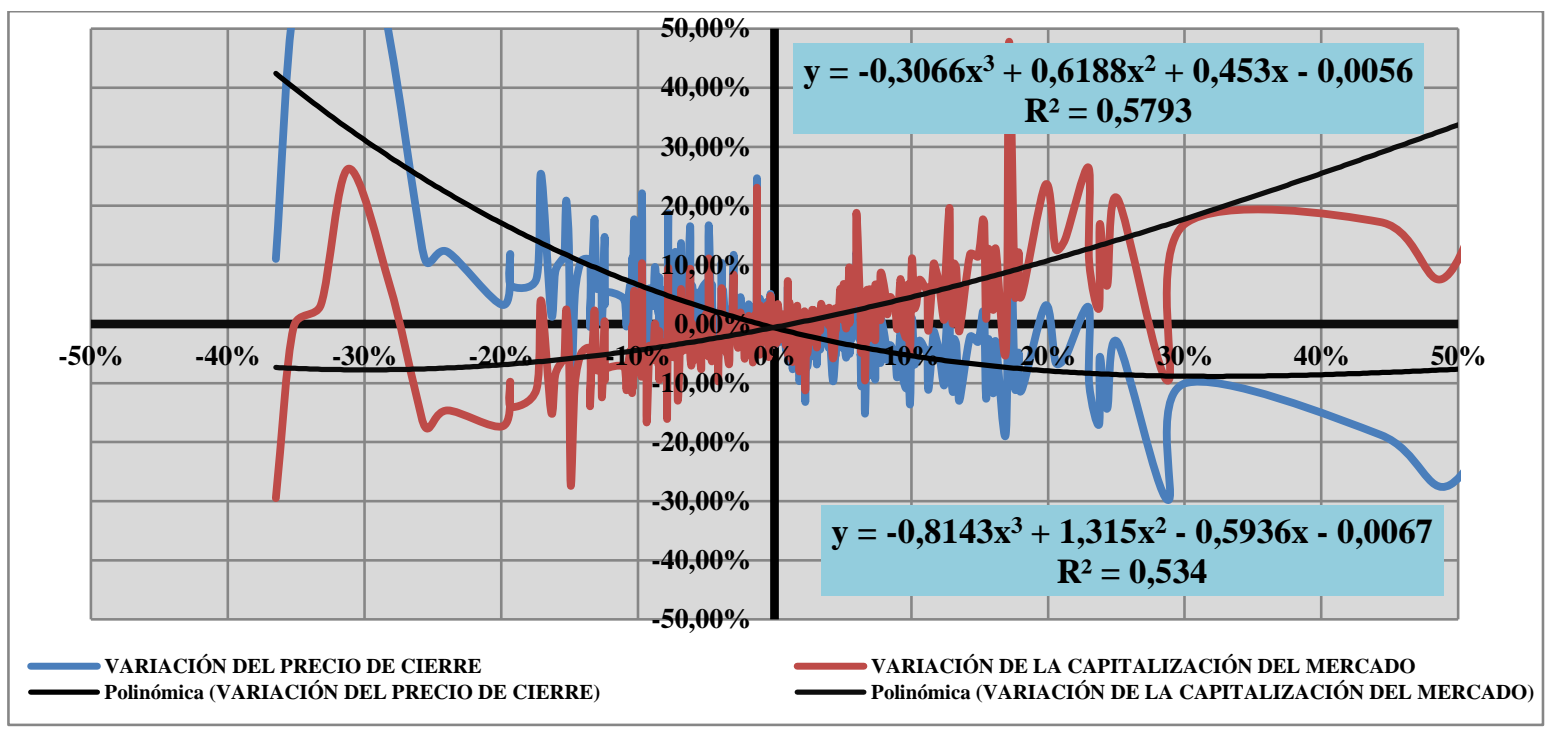

Figura 8. Variación del indicador bursátil (índice bursátil) respecto al precio de cierre y la capitalización del mercado del Ripple.

Fuente: Elaboración propia en base a datos de coinmarketcap.com

En las figuras 7 y 8 , se observa como la variaciones de la capitalización del mercador respecto al índice bursátil muestran una curva ascendente, mientras que respecto al precio de cierre una curva descendente, es decir a un incremento del índice bursátil, se tiene una variación positiva de la capitalización del mercado y una variación negativa respecto al precio de cierre; mientras ante una disminución del índice bursátil, se tiene un incremento del precio de cierra y una disminución del precio del mercado. 
Por ello para mantener un equilibrio entre la variación del precio de cierre y la capitalización del mercado la misma red hace un ajuste cada 24 horas del incrementando o no la capitalización del mercado en función de la variación del precio de cierre del día anterior. Por ello un aumento del indicador bursátil (capitalización/precio) se traducirá en una disminución de la tasa de rendimiento de la inversión vía precios más bajos, y con ello una menor eficiencia marginal del capital invertido en criptomonedas, ello sin duda alguna coadyuva a contrarrestar los ataques especulativos del mercado.

Finalmente las figuras 9 y 10, se proyectan ambas curvas mostradas en las figuras 7 y 8 ; es decir, la curva ascendente como variable independiente y la curva descendente como variable dependiente en aras de determinar como la variación de la capitalización respecto al índice bursátil, se comporta respecto a la variación del precio de cierre respecto al índice bursátil, lo cual deja entre ver otro elemento probatorio de la ley de los rendimiento marginales decrecientes, como parte componente para evaluar la eficiencia marginal del capital; es decir un aumento de la criptomonedas sin un crecimiento correspondiente del nivel de capitalización en el mercado, provocara una disminución del precio de los criptoactivos.

De acuerdo a las criptomonedas analizadas en el presente estudio, en las figuras 8 y 9 , se muestra que en el caso del Bitcoin su precio de cierre, puede incrementarse más de un $15 \%$ si la capitalización del mercado se contrae entre un $20 \%$ y un $15 \%$ o disminuir hasta un $10 \%$, si este aumenta entre un $10 \%$ y $20 \%$; mientras en el caso del Ripple este podría crecer hasta un $40 \%$ y disminuir hasta un $10 \%$ si la capitalización del mercado se contrae entre un $10 \%$ y un $5 \%$ o disminuir hasta un $10 \%$, si este aumenta entre un $10 \%$ y $20 \%$; lo cual es totalmente concordante con la teoría de la ley de los rendimientos marginales decrecientes, antes señalada.

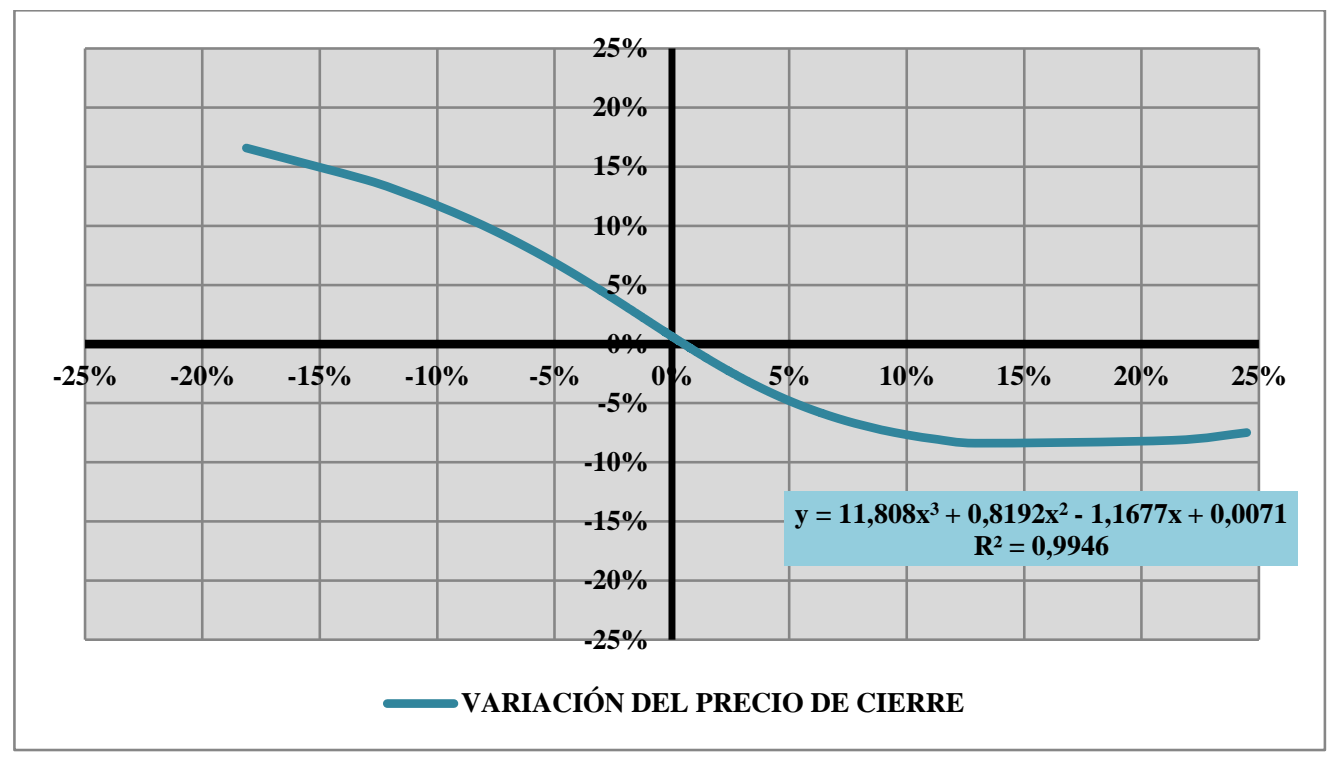

Figura 9. Variación del precio de cierre respecto a la variación de la capitalización del mercado en el Bitcoin

Fuente: Elaboración propia en base a datos de coinmarketcap.com 


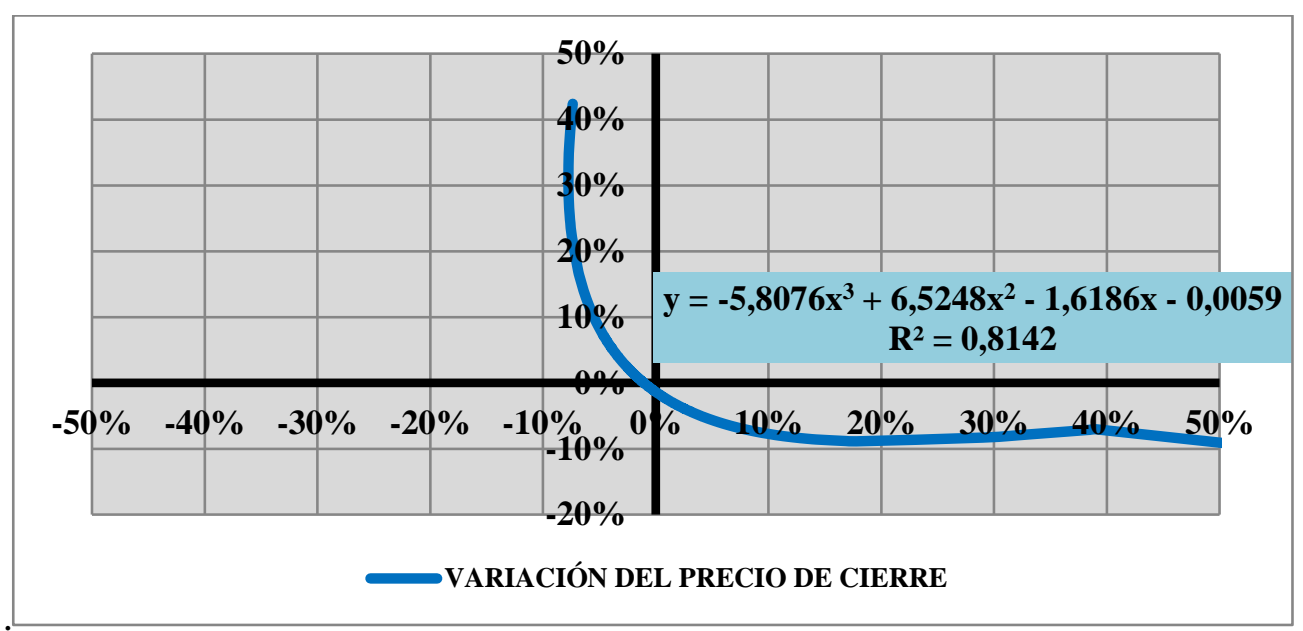

Figura 10. Variación del precio de cierre respecto a la variación de la capitalización del mercado en el Ripple

Fuente: Elaboración propia en base a datos de coinmarketcap.com

\section{Conclusiones.}

- Luego de haber analizado las dos criptomonedas minadas y nominadas de mayor cotización en el mercado de los criptoactivos, puede concluirse que las mismas se ajustan a los preceptos de la que contemplan la eficiencia marginal del capital en lo referente a la ley de los rendimientos marginales decrecientes y la productividad marginal del capital. Sumado a ello se evidencia el ajuste diario con un rezago de 24 horas que se realiza de la capitalización en el mercado de las criptomonedas con respecto al precio de cierre. Como existe una variación inversa de la capitalización en el mercado con respecto a la variación de precio de cierre en ese período de rezago intertemporal.

- En el estudio se pudo constatar en el caso del Bitcoin el crecimiento exponencial de su precio en el mercado al superar en la primera quincena de octubre de 2017 la banda de resistencia de los $\$ 5.000$ y alcanzar un máximo valor de $\$ 19.497,40$ a mediados diciembre de 2017, para luego comenzar una caída o evolución con pendiente negativa de su precio de cierre hasta finales de junio de 2018 llegando a su cotización más baja de $\$ 5.903,44$ para luego comenzar a crecer nuevamente, por lo que puede afirmarse que en el segundo trimestre de 2018, el precio de cierre del Bitcoin ha presentado como banda de soporte los $\$ 5.000$ y como banda de resistencia los $\$ 10.000$; esto hace proyectar en el mejor de los casos un nuevo auge de crecimiento de precios en el último trimestre del año, o una estabilidad de precios en lo que resta del año 2018.

- En tanto que el Ripple tuvo un crecimiento exponencial del nivel de precio de cierre del mercado a mediados de diciembre de 2017, superando la banda de resistencia de los 40 centavos de dólar, hasta al alcanzar su tope de precio a inicios de enero de 2018 de $\$ 3,38$ posteriormente producirse una caída o evolución con pendiente negativa de su precio de cierre hasta finales de junio de 2018 llegando a su cotización más baja de 45 centavos de dólar para luego comenzar a crecer nuevamente, por lo que se puede aseverar que en el segundo trimestre de 2018, el precio de cierre del Ripple ha presentado como banda de soporte los 40 centavos 
de dólar y como banda de resistencia \$1; lo que hace proyectar en el mejor de los casos un nuevo auge de crecimiento de precios en el último trimestre del año, o una estabilidad de precios en lo que resta del año 2018.

\section{Referencias Bibliográficas.}

Guerrero , G., \& Guerrero, M. (2014). Metodología de la Investigación. México: Grupo Editorial Patria.

Konkin, S. (1983). New Libertarian Manifesto. Los Angeles: Koman Publishing.

Laurence, T. (2017). Blockchain For Dummies. New York: Wiley-Blackwell.

Antonopoulos, A. (2015). Mastering Bitcoin: Unlocking Digital Cryptocurrencies. Sebastopol: O'Reilly Media, Inc.

Bernal, C. (2006). Metodología de la investigación: para administración, economía, humanidades y ciencias sociales. México: Pearson Educación.

Draper, J. (2013). Is bitcoin the future of money? The Straight Dope.

Gaviria, F. (2006). Moneda, Banca y Teoría Monetaria. Bogotá: Fundación Universidad de Bogotá.

Guttmann, B. (2014). The Bitcoin Bible Gold Edition: All you need to know about bitcoins and more. BoD-Books on Demand.

Hernández, R., Fernández, C., \& Baptista, P. (2014). Metodología de la investigación. México: McGraw Hill Educación.

Hicks, J. (1962). Liquidity. Economic Journal, 787-802.

Huerta de Soto, J. (2004). Estudios de economía política. Madrid: Unión editorial.

Kane, E. (1981). Accelerating Inflation, Technological Innovation, and the Decreasing Effectiveness of Bank Regulation. Journal of Finance, 36 , 355-367.

Keynes, J. (2014). Teoría general de la ocupación, el interés y el dinero. México: Fondo de Cultura Económica.

Krugman, P. (2013, Abril 19). La red antisocial de los bitcoins. El País.

Markowitz, H. (1952). Portfolio selection. The journal of finance, 7(1), 77-91.

Markowitz, H. (1970). Portfolio Selection: Efficient Diversification of Investments (New York, 1959). New York: JOHN WILEY \& SONS, INC.

Mishkin, F. (2008). The economics of money, banking and financial markets. Toronto: Pearson Canada. 
Norman, A. (2017). Mastering Bitcoin for Starters: Bitcoin and Cryptocurrency Technologies, Mining, Investing and Trading. Create Space Independent Publishing Platform.

Pérez, R. (2011). Teoría y práctica de la bolsa: Todo lo que debe saber el inversor sobre los mercados financieros. Madrid: Ediciones Díaz de Santos.

Ramirez, E. (2007). Moneda, Banca y Mercados Financieros: Instituciones e Instrumentos en Paises en Desarrollo. México: Pearson Educación.

Reid, F., \& Harrigan, M. (2013). An analysis of anonymity in the bitcoin system. In Security and privacy in social networks. Springer,, 197-223.

Rogojanu, A., \& Badea, L. (2014). The issue of competing currencies. Theoretical \& Applied Economics, 22(2).

Roy, A. (1952). Safety first and the holding of assets. Econometrica, 20 (3), 431-449.

Samuelson, P., \& Nordhaus, W. (2006). Microeconomía. Madrid: McGraw-Hill Interamericana.

Sharpe, W. (1963). Simplified Model for Portfolio Analysis. . Management Science. Volume 9, №. 2 (January), : 277-293. .

Yermack, D. (2013). Is Bitcoin a Real Currency? An economic appraisal. National Bureau of Economic Research. 


\section{Para citar el artículo indexado.}

Urdaneta A., Borgucci E., Campuzano J. \& Avilés P. (2019). Hechos estilizados del mercado de criptomonedas desde el enfoque de la eficiencia marginal del capital. Revista electrónica Visionario Digital 3(2), 228-244. Recuperado desde: http://visionariodigital.org

\section{Liencia}

El artículo que se publica es de exclusiva responsabilidad de los autores y no necesariamente reflejan el pensamiento de la Revista Ciencia Digital.

El articulo queda en propiedad de la revista y, por tanto, su publicación parcial y/o total en otro medio tiene que ser autorizado por el director de la Revista Ciencia Digital.
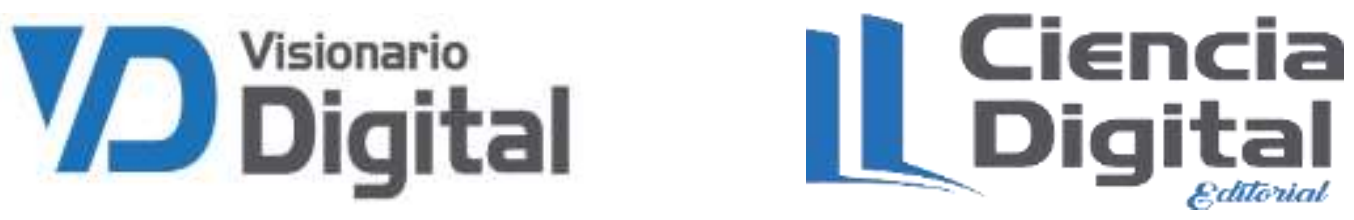